\title{
Human antibody response to Zika targets type-specific quaternary structure epitopes
}

Matthew H. Collins, ${ }^{1,2}$ Huy A. Tu, ${ }^{3,4}$ Ciara Gimblet-Ochieng, ${ }^{5}$ Guei-Jiun Alice Liou, ${ }^{5}$ Ramesh S. Jadi, ${ }^{5}$ Stefan W. Metz, ${ }^{5}$ Ashlie Thomas, ${ }^{5}$ Benjamin D. McElvany, ${ }^{4}$ Edgar Davidson, ${ }^{6}$ Benjamin J. Doranz,${ }^{6}$ Yaoska Reyes, ${ }^{7}$ Natalie M. Bowman, ${ }^{2}$ Sylvia Becker-Dreps, ${ }^{8}$ Filemón Bucardo, ${ }^{7}$ Helen M. Lazear, ${ }^{5}$ Sean A. Diehl, ${ }^{3,4}$ and Aravinda M. de Silva ${ }^{5}$

'Department of Medicine, Emory University, Atlanta, Georgia, USA, and Hope Clinic of the Emory Vaccine Center, Division of Infectious Diseases, Department of Medicine, School of Medicine, Emory University, Decatur, Georgia, USA. 2Department of Medicine, Division of Infectious Diseases, University of North Carolina School of Medicine, Chapel Hill, North Carolina, USA. ${ }^{3}$ Cellular, Molecular, and Biomedical Sciences Program, University of Vermont, Burlington, Vermont, USA. "Vaccine Testing Center, Department of Microbiology and Molecular Genetics, Larner College of Medicine, University of Vermont, Burlington, Vermont, USA. ${ }^{5}$ Department of Microbiology and Immunology, University of North Carolina School of Medicine, Chapel Hill, North Carolina, USA. ${ }^{6}$ Integral Molecular, Inc., Philadelphia, Pennsylvania, USA. 'Department of Microbiology, Faculty of Medical Sciences, National Autonomous University of León, Nicaragua. ${ }^{8}$ Departments of Family Medicine and Epidemiology, University of North Carolina at Chapel Hill, Schools of Medicine and Public Health, Chapel Hill, North Carolina, USA.

The recent Zika virus (ZIKV) epidemic in the Americas has revealed rare but serious manifestations of infection. ZIKV has emerged in regions endemic for dengue virus (DENV), a closely related mosquito-borne flavivirus. Cross-reactive antibodies confound studies of ZIKV epidemiology and pathogenesis. The immune responses to ZIKV may be different in people, depending on their DENV immune status. Here, we focus on the human B cell and antibody response to ZIKV as a primary flavivirus infection to define the properties of neutralizing and protective antibodies generated in the absence of preexisting immunity to DENV. The plasma antibody and memory B cell response is highly ZIKV type-specific, and ZIKV-neutralizing antibodies mainly target quaternary structure epitopes on the viral envelope. To map viral epitopes targeted by protective antibodies, we isolated 2 type-specific monoclonal antibodies (mAbs) from a ZIKV case. Both $\mathrm{mAbs}$ were strongly neutralizing in vitro and protective in vivo. The $\mathrm{mAbs}$ recognize distinct epitopes centered on domains I and II of the envelope protein. We also demonstrate that the epitopes of these mAbs define antigenic regions commonly targeted by plasma antibodies in individuals from endemic and nonendemic regions who have recovered from ZIKV infections.

Authorship note: MHC and HAT contributed equally to this work.

Conflict of interest: MHC, HT, BDM, $S A D$, and AMDS are co-inventors on a patent application (US 62589006, filed 11/21/2017) describing the discovery and use of A9E and G9E human mAbs. BD and ED are employees of Integral Molecular.

Copyright: (c) 2019 American Society for Clinical Investigation

Submitted: September 4, 2018

Accepted: March 7, 2019

Published: April 18, 2019

Reference information: /CI Insight. 2019;4(8):e124588. https://doi. org/10.1172/ji.insight.124588.

\section{Introduction}

Zika virus (ZIKV) became a prominent international concern in 2015 when it caused a large epidemic in the Americas linked to thousands of birth defects, miscarriages, and stillbirths, as well as cases of Guillain-Barre syndrome in several countries (1). These severe phenotypes are in contrast to the majority of infections that are asymptomatic or cause only a self-limited illness. ZIKV is only one example of many expanding, emerging, or reemerging mosquito-borne flaviviruses (2). In locales where yellow fever vaccine is not routinely administered, sporadic outbreaks result in 100,000 severe cases, with tens of thousands of deaths each year (3), and dengue virus (DENV) continues to pose a threat to two-thirds of the world's population, with more than 300 million new infections every year (4). To successfully deal with emerging flaviviruses, multifaceted and coordinated response efforts are required. Central to these endeavors is a comprehensive knowledge of human immune responses to these pathogens, which directly supports development of vital public health tools such as vaccines and diagnostics. Indeed, vaccination against yellow fever, tick-borne encephalitis, and Japanese encephalitis has demonstrated the potential public health benefit of developing effective vaccines against flaviviruses (4). 
ZIKV has an 11-kb positive-sense, single-stranded RNA genome, which encodes 7 nonstructural (NS) proteins and 3 structural proteins: capsid (C), premembrane (prM), and envelope (E) (1). Although other components of the adaptive immune system such as $\mathrm{T}$ cells are likely important for long-term immunity ( 5 , 6), a large body of work has supported a central role for neutralizing antibody $(\mathrm{Ab})$ responses. E protein is the main target of neutralizing and protective Abs elicited in people exposed to flavivirus infections or vaccines (7-14). The flavivirus E protein is a class II viral fusion protein that mediates attachment to cellular receptors and low-pH-triggered fusion within endosomes required for viral entry into cells. The $\mathrm{E}$ protein monomer contains 3 distinct domains, designated EDI, EDII, and EDIII (15). The surface of the flavivirus virion is covered by $90 \mathrm{E}$ protein homodimers, which are tightly packed to form a viral envelope with icosahedral symmetry $(16,17)$. For DENV and West Nile virus, flaviviruses closely related to ZIKV, human neutralizing Abs often target complex or quaternary epitopes, with Ab binding footprints that include residues on multiple adjacent $\mathrm{E}$ monomers on the intact virion (18-21).

Particularly for the 4 DENV serotypes, studies have demonstrated that humans exposed to primary flavivirus infections develop type-specific neutralizing Abs and memory B cells (MBCs) that are strongly correlated with long-term protection from reinfection by the same virus $(12,22,23)$. Understanding humoral immunity to ZIKV is complicated, in that most ZIKV transmission occurs in areas where DENV (and potentially other flaviviruses) are endemic, with DENV seroprevalence as high as $90 \%$ by early adulthood $(24,25)$. Ab cross-reactivity at the level of binding and neutralization is a well-known phenomenon among flaviviruses in general and between DENV and ZIKV in particular, which can confound serologic assays (26-29). The impact of Ab cross-reactivity on clinical outcomes for ZIKV infections in DENV-immune hosts (and vice versa) remains an active area of investigation (30-32). Extensive cross-reactivity is expected given considerable conservation in the amino acid sequences of DENV and ZIKV E (approximately 50\%) $(17,33)$. Furthermore, B cell and Ab responses to a second DENV infection are skewed by preferential activation of preexisting cross-reactive MBCs. Indeed, recent studies suggest that a similar phenomenon may occur when ZIKV infects a DENV-immune person (34-37). However, we (38) and others $(35,36)$ have observed that ZIKV type-specific Ab responses develop in humans even in the presence of immunity to prior DENV infection.

Here, we focused on understanding the molecular determinants of the human neutralizing Ab response to primary ZIKV infection. To date, most ZIKV infections have occurred in DENV-immune individuals, but as ZIKV becomes endemic throughout Latin America and the Caribbean, more individuals will experience ZIKV as their first flavivirus infection. To learn how ZIKV may affect immunity to subsequent flavivirus infection, or how secondary flavivirus infection could alter existing immunity to ZIKV, we must first understand the immune response to ZIKV in the absence of other (particularly DENV) flavivirus exposures. Additionally, understanding the properties and mechanisms underlying the induction of neutralizing Abs in naive individuals is directly relevant to developing vaccines for use in flavivirus-naive populations living in countries with endemic DENV and ZIKV transmission.

\section{Results}

Human subjects with primary $Z I K V$. To understand the $\mathrm{Ab}$ response when ZIKV is a primary flavivirus infection, we identified 4 US residents that acquired ZIKV infection during foreign travel (Table 1). All infections were in Latin America from 2015 to 2016 and experienced uncomplicated, self-limited, symptomatic infections that resolved within 1 week. As we were mainly interested in the long-term $\mathrm{MBC}$ and plasma $\mathrm{Ab}$ responses, only late-convalescence blood samples collected 6 months or more after infection were analyzed. Subjects DT206 and DT244 reported positive ZIKV PCR testing during clinical evaluation following travel, and we detected anti-ZIKV IgM in plasma samples obtained from those 2 subjects within 12 weeks of infection but not in the late-convalescence samples used in these studies (data not shown). All 4 subjects had strong type-specific neutralizing $\mathrm{Ab}$ responses to $\mathrm{ZIKV}$ (50\% focus reduction neutralization test $\left[\mathrm{FRNT}_{50}\right]$ titer range 1,845-5,267) (Table 1 and Figure 1C).

Human Ab responses to primary Zika. Plasma from all 4 ZIKV cases exhibited positive IgG binding in antigen-capture ELISA using ZIKV or a mix of DENV1-4 virus as antigens, over a range of plasma dilutions (Figure 1A). The binding signal for DENV decayed more rapidly than for ZIKV, reaching assay limit of detection between the 1:500 and 1:1000 dilution. IgG binding to ZIKV was readily detectable over background for all 4 primary ZIKV plasma at 1:1000, indicating higher IgG titers against ZIKV. All 4 plasma samples contained IgG Abs that also bound ZIKV recombinant E (ZIKV E80) and E domain I and III 
Table 1. Demographic and serologic characteristics of subjects

\begin{tabular}{|l|c|c|c|c|c|c|c|c|}
\hline \hline & \multirow{2}{*}{$\begin{array}{c}\text { Place } \\
\text { infected }\end{array}$} & Time after infection & \multirow{2}{*}{ YFV vaccine } & \multirow{2}{*}{ Symptoms } & & \multicolumn{3}{|c|}{ FRNT $_{\mathbf{5 0}}$} \\
\cline { 6 - 10 } & & & DENV1 & DENV2 & DENV3 & DENV4 & ZIKV \\
\hline DT168 & Brazil & 6 months & Unknown & F, R, C, HA, AR, GI & $<20$ & $<20$ & 28 & $<20$ \\
\hline DT172 & Colombia & 6 months & No & F, R, C, AR & $<20$ & $<20$ & $<20$ & $<20$ \\
\hline DT206 & Honduras & 6 months & No & F, R, HA, AR & $<20$ & $<20$ & $<20$ & $<20$ \\
\hline DT244 & Puerto Rico & 6 months & No & R & $<20$ & $<20$ & $<20$ & $<20$ \\
\hline
\end{tabular}

F, fever; R, rash; C, conjunctivitis; HA, headache; AR, arthritis/arthralgia; GI, nausea, vomiting, or diarrhea.

(ZVEDI and ZVEDIII) (Figure 1B). Consistent with the serologic diagnostic criteria used here to confirm ZIKV cases, the plasma from these 4 travelers strongly neutralized ZIKV by FRNTs and exhibited minimal to no cross-neutralization of DENV serotypes 1-4 (Table 1 and Figure 1C).

Contribution to neutralization activity by distinct Ab subsets. Strongly neutralizing Ab responses to DENV often target $\mathrm{E}$ protein quaternary epitopes displayed on the virion but not on recombinantly expressed monomeric E protein (18-20). We tested whether plasma neutralizing Abs in people infected with ZIKV recognized simple or quaternary epitopes on E protein. A recombinantly expressed monomeric ectodomain of ZIKV E protein (ZIKV E80) was purified, immobilized onto beads, and used to deplete all ZIKV E80-binding Abs from the plasma of primary ZIKV cases. Confirmation ELISA demonstrated loss of ZIKV E80 binding activity (Figure 2A), but retained IgG binding to intact virions (Figure 2B), albeit at variably reduced levels compared with the undepleted specimens. Compared with control depleted plasma, neutralization activity of ZIKV E80-depleted plasma was unaffected for DT172 and

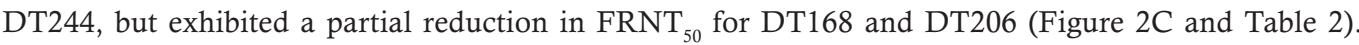
To confirm that the majority of ZIKV neutralization is attributable to Abs against quaternary epitopes, plasma from each of the 4 primary ZIKV cases were depleted with ZIKV virus-like particles (VLPs), which present all conformational epitopes of the intact virion. Marked loss of neutralization activity was observed (Figure 2D and Table 2). Taken together, these results indicate that primary ZIKV infection elicits a complex $\mathrm{Ab}$ response that includes populations of $\mathrm{Abs}$ that are cross-reactive but non-neutralizing to DENV, as well as ZIKV type-specific neutralizing Abs. Furthermore, ZIKV-neutralizing Abs target quaternary epitopes, though a minor fraction of neutralizing activity is attributable to Abs that target epitopes on the $\mathrm{E}$ monomer in some individuals.

The $M B C$ response to primary ZIKV infection. To assess the $\mathrm{MBC}$ repertoire in primary ZIKV infection, we immortalized MBCs (39) from DT168 and DT172. Screening supernatants of polyclonal cultures of sorted immortalized MBCs for IgG binding to ZIKV and DENV1-4 revealed the frequencies of antigen-specific MBCs (Figure 3). This calculation was based on the assumption of stochastic sampling during sorting and the average presence of 1 unique clone in the originating 50-cell polyclonal culture capable of producing IgG that yielded a positive ELISA signal. Across 480 polyclonal cultures (24,000 MBC clones), 267 cultures were ZIKV reactive. The vast majority of these culture supernatants showed exclusive specificity for ZIKV ( $n=222,83 \%$ ), and a minority were cross-reactive to ZIKV and DENV ( $n=45,17 \%$ ) (Figure 3 ). For DT168, the frequency of ZIKV-specific MBCs was $1.2 \%$ of total MBCs, with $1 \%$ ZIKV type-specific and $0.2 \%$ ZIKV/DENV cross-reactive. DT172 was similar, with $0.9 \%$ ZIKV-reactive MBCs, comprising $0.8 \%$ ZIKV type-specific and $0.1 \%$ ZIKV/DENV cross-reactive.

Potent ZIKV-specific neutralizing monoclonal Abs following primary ZIKV infection. To better understand the molecular determinants of ZIKV neutralization, we sought to isolate neutralizing monoclonal Abs (mAbs) and use these as tools for more-detailed studies of virion-Ab interactions. Using single-cell sorting, we established monoclonal MBC cultures from 10 polyclonal cultures with positive ZIKV ELISA binding signals for subject DT168 (Table 3). We recovered approximately 40\% of the single-cell cultures as proliferating, IgG-producing cultures. Supernatants from monoclonal cultures were then screened for ZIKV-specific IgG. Half of the polyclonal cultures yielded ZIKV-reactive monoclonal cultures. Among monoclonal cultures from a given polyclonal progenitor culture, we found multiple positive wells. For some monoclonal cultures (e.g., E3, H10, and G11), all of the positive wells exhibited an extremely narrow range of values for ZIKV binding (as assessed by optical density [OD] in ELISA), suggesting clonality, which for E3 was confirmed by sequencing all $6 \mathrm{ZIKV}$-specific subclones. This is consistent with the assumption that 1 clone 

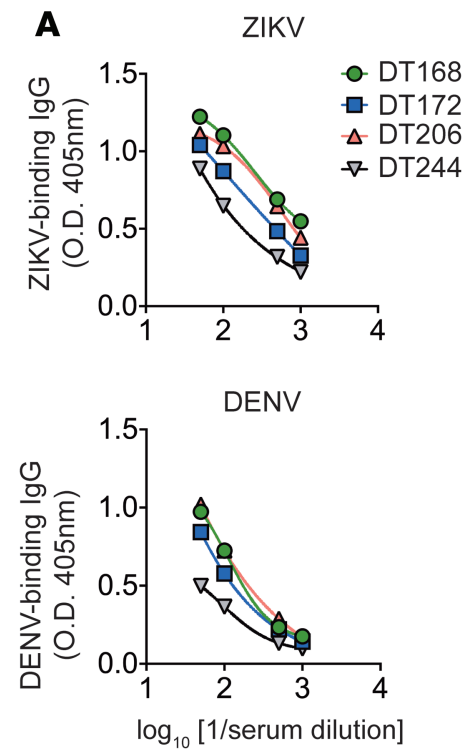

B

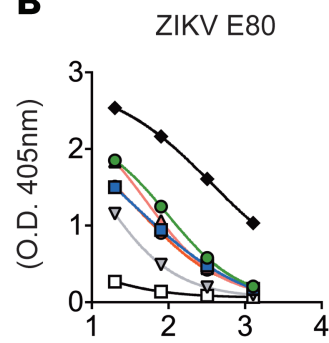

ZIKV EDI

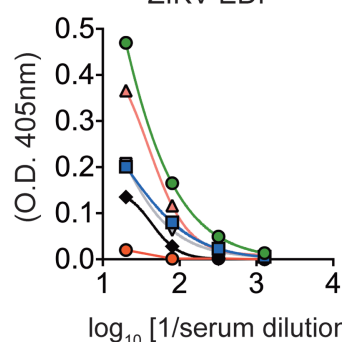

DENV E80

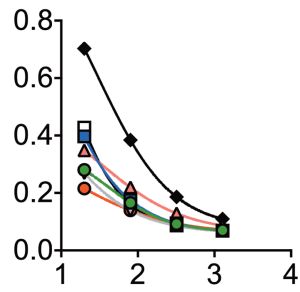

- $\mathrm{DT} 172$

$\triangle$ DT206

$\nabla$ DT244

^ DT000

- DT001

- ㅁ NHS

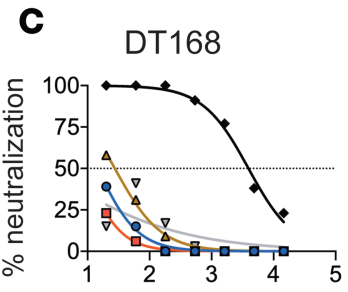

$\log _{10}[1 /$ serum dilution]

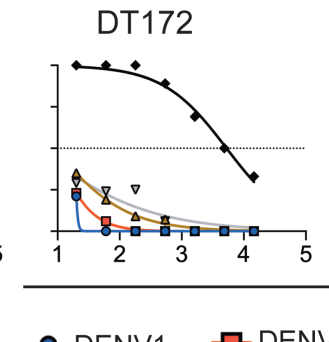

DT206

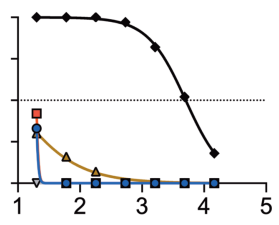

DT244

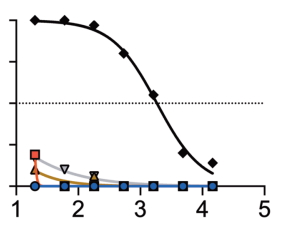

$2^{\circ}$ DENV

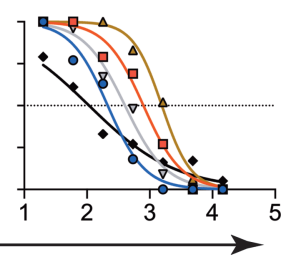

DENV1

DENV2

$\triangle$ DENV3

DENV4

ZIKV

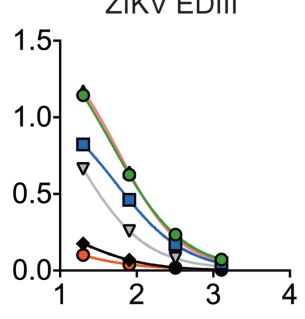

Figure 1. Primary serologic response to ZIKV. (A) Plasma from 4 primary ZIKV cases (DT168, DT172, DT206, and DT244) were tested for IgG binding to ZIKV (top) and DENV (bottom) over the dilution series indicated on the $x$ axis. (B) Primary ZIKV plasma and primary (DTO01) and secondary (DTO0O) DENV plasma were tested for IgC binding to ZIKV recombinant E (ZIKV E80), DENV recombinant E (DENV E80), ZVEDI, and ZVEDIII. (C) Neutralization assays were also performed for each primary ZIKV plasma as well as a secondary $\left(2^{\circ}\right)$ DENV control. NHS, naive human plasma (negative binding control for ELISA).

in the original polyclonal culture was responsible for the initial positive signal. For E4- and D1-derived clones, positive monoclonal wells fell into 2 categories of OD values differing by more than $20 \%$, each with very narrow $(<10 \%)$ intragroup OD value range, suggesting 2 clones may have been present in the polyclonal culture. We confirmed this for D1 by sequencing 1 of the 2 potential clones and found 2 identical subclones of the G9E monoclonal culture. Taken together, these results support our assumption that, on average, 1 clone in the 50-cell polyclonal culture produces a positive signal, thus validating the calculation for estimating ZIKV-specific MBC frequencies as above. Occasionally, multiple reactive clones may exist in the polyclonal culture. This would be more likely to happen when the frequency of antigen-specific MBCs is higher, and it would lead to potential underestimation of the antigen-specific MBC frequency. ZIKV IgG-positive reactive supernatants were then screened for neutralization activity (Supplemental Figure 1; supplemental material available online with this article; https://doi.org/10.1172/jci.insight.124588DS1; and data not shown). Again, subclones with tight $O D$ values exhibited near-identical neutralization values.

We then isolated RNA from monoclonal cultures producing the 2 most potent ZIKV-neutralizing mAbs (A9E and G9E) and assessed IgG isotype, heavy chain-light chain pairing, V gene usage, complementarity-determining region 3 (CDR3) sequence, and somatic hypermutations (SHMs) by sequencing of Ig heavy and light chain gene products (Table 4 ) as described previously (40). We recovered 2 distinct mAbs - both were IgG1, both used Ig- $\lambda$ light chain, and exhibited a high level of non-silent SHMs in their CDR regions compared with framework regions (FWRs) across IGH and IGL. The $2 \mathrm{mAbs}$ were distinct in heavy chain $\mathrm{V}(\mathrm{D}) \mathrm{J}$ gene usage and CDR3 sequence. These unique $\mathrm{mAb}$ IGH and IGL sequences were inserted into IgG1/Ig- $\lambda$ expression vectors, respectively, and produced in HEK-293F cells as described previously $(40,41)$. 
A

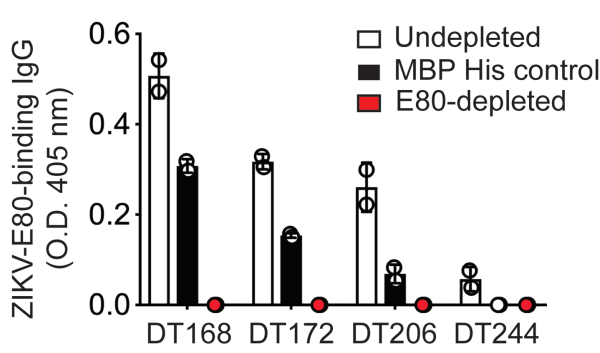

B

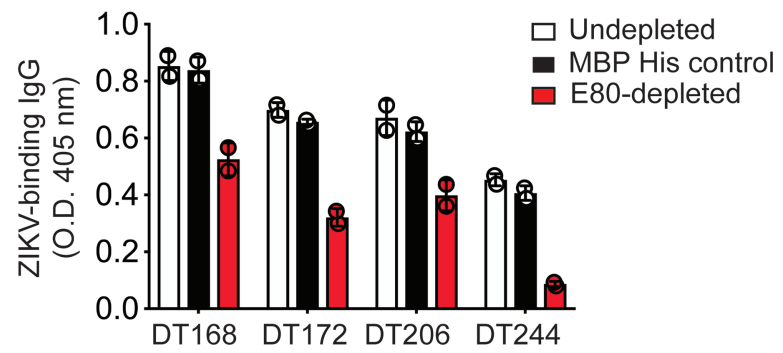

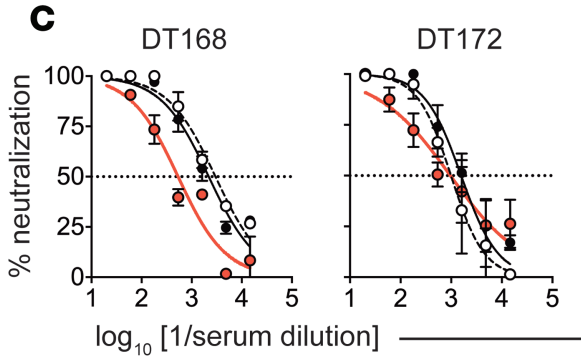
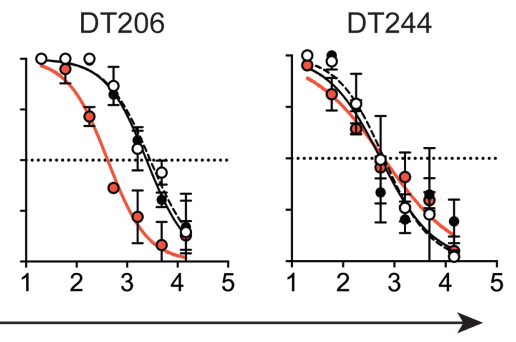

$$
\begin{aligned}
& \text {--o-- Undepleted } \\
& \rightarrow-\text { MBP His control } \\
& \rightarrow-\text { E80-depleted }
\end{aligned}
$$

D

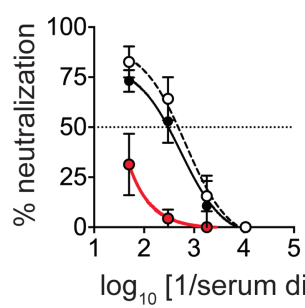

DT172

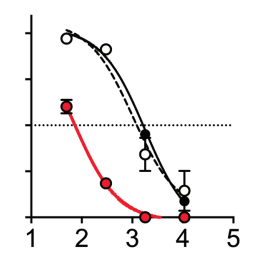

DT206

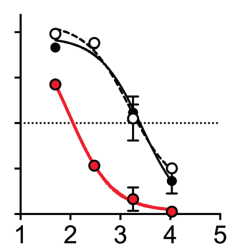

DT244

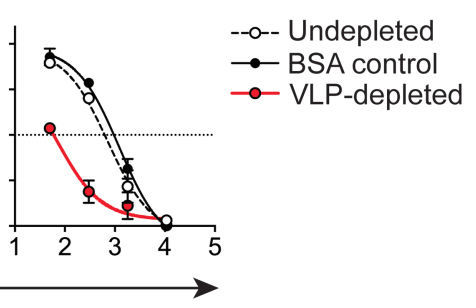

Figure 2. Antibodies against quaternary epitopes are the predominant mediators of ZIKV neutralization. (A) Depletion of ZIKV E80-binding IgG in primary ZIKV plasma was confirmed by direct antigen-coating ELISA comparing ZIKV E80-binding IgG in depleted (red bars) to MBP-control-depleted (white bars) or undepleted (black bars) plasma. Mean optical density values from flavivirus-uninfected plasma (background) are subtracted from each group. (B) IgG binding to ZIKV in depleted plasma was tested by antigen-capture ELISA. Mean optical density values from flavivirus-uninfected plasma (background) are subtracted from each group. (C) FRNT assays were performed for ZIKV E80-depleted plasma and controls using ZIKV H/PF/2013. (D) FRNT assays were performed for ZIKV VLP-depleted plasma and controls using ZIKVH/PF/2013.

Binding dynamics and epitope mapping. Both the A9E and G9E human mAbs bound ZIKV virions in an antigen-capture ELISA, but did not bind to DENV4, which was tested as a representative DENV serotype (Figure 4A). This result agreed with the initial characterization of the polyclonal cultures from which these mAbs were derived. Surprisingly, both mAbs bound to recombinant ZIKV E80, and A9E bound to ZVEDI $\left(\mathrm{EC}_{50}=2,500 \mathrm{ng} / \mathrm{ml}\right)$, albeit at higher concentrations compared with ZIKV E80 $\left(\mathrm{EC}_{50}=40 \mathrm{ng} / \mathrm{ml}\right)$. Neither $\mathrm{mAb}$ bound to ZVEDIII (Figure 4B). Both mAbs were unable to bind DENV1-4 when testing each serotype individually, confirming ZIKV specificity (Supplemental Figure 2). To approximate the location of the epitope recognized by each $\mathrm{mAb}$, competition assays were performed (hereafter referred to as blockade of binding [BOB]). A panel of 6 flavivirus cross-reactive and $6 \mathrm{ZIKV}$-specific mAbs were competed with A9E and G9E in BOB assays. DENV-specific mAbs were used as a control to establish $100 \%$ binding. As a positive control, unlabeled A9E or G9E mAb was competed with itself and showed a high level of autoblockade (Figure 4C). None of the DENV type-specific controls decreased the OD signal of A9E or G9E binding compared with control. Most flavivirus cross-reactive $\mathrm{mAbs}$ and $\mathrm{ZIKV}$-specific $\mathrm{mAbs}$ failed to appreciably reduce the binding of A9E or G9E, with 2 notable exceptions. Both EDE1 mAbs C8 and C10 (42), which bind across domain II of E molecules paired in a homodimer, showed partial blockade of G9E. Additionally, ZKA190, a human ZIKV-specific mAb known to bind to the EDI-III linker and lateral ridge of EDIII (43), strongly blocked A9E with an $\mathrm{EC}_{50}$ similar to that exhibited by A9E against itself. Neither of these $2 \mathrm{mAbs}$ exhibited BOB activity against the other (data not shown), indicating that the $2 \mathrm{mAbs}$ target distinct, non-overlapping epitopes.

Both A9E and G9E strongly neutralized Asian and African lineages of ZIKV (Figure 4, D and E, and Supplemental Figure 3), but exhibited no activity against any of the 4 DENV serotypes, St. Louis encephalitis 
Table 2. Neutralization titers in Zika-immune plasma following ZIKV antigen depletion

\begin{tabular}{|c|c|c|c|c|c|c|}
\hline & Undepleted & Control & ZIKV E80 & Undepleted & Control & ZIKV VLP \\
\hline DT168 & 2,985 & 2,191 & 535 & 676 & 538 & $<50$ \\
\hline DT172 & 992 & 1,572 & 964 & 1,023 & 1,787 & 70 \\
\hline DT206 & 2,884 & 2,473 & 413 & 1,513 & 2,742 & 94 \\
\hline DT244 & 630 & 538 & 617 & 717 & 1,218 & 73 \\
\hline
\end{tabular}

Numbers are FRNT $_{50}$ values expressed as the calculated plasma dilution factor.

virus, or yellow fever virus. A9E and G9E exhibited mean FRNT $_{50}$ concentrations of 8.3 and $29 \mathrm{ng} / \mathrm{ml}$ across all ZIKV strains tested. Finally, to investigate the potential of these mAbs to protect against ZIKV infection in vivo, we treated 5-week-old Ifnar ${ }^{-1}$ mice (44) with $200 \mu \mathrm{g}$ of A9E, G9E, or isotype control IgG by intraperitoneal injection 1 day prior to subcutaneous foot pad infection with 1,000 focus-forming units (FFU) ZIKV (H/ $\mathrm{PF} / 2013)$. Control mice lost weight and succumbed to infection by $8-10$ days, whereas all mice that received A9E or G9E gained weight and did not succumb to infection (Figure 4, F and G).

Escape mutations and further epitope mapping. To investigate the determinants for neutralizing $\mathrm{mAb}$ binding in a biologically relevant model system, ZIKV (PRVABC59) was passaged under increasing concentrations of A9E and G9E mAbs on Vero cells. No escape virus that could tolerate increasing concentrations of G9E was isolated, even when beginning the process with a concentration of G9E as low as $20.6 \mathrm{ng} / \mathrm{ml}$. In contrast, for $\mathrm{A} 9 \mathrm{E}$, an escape virus was isolated after 3 rounds of passage that could be propagated in the presence of 35,800 $\mathrm{ng} / \mathrm{ml} \mathrm{A9E} \mathrm{mAb} \mathrm{(approximately} 780 \times \mathrm{FRNT}_{50}$ ). Viral isolates were plaque purified to generate clonal stocks. Two viral isolates were tested for binding by $\mathrm{mAb}$ and plasma (Figure $5 \mathrm{~A}$ ) and for neutralization escape (Figure $5 \mathrm{~B}$ ). Isolate nomenclature is as follows: passage 4 from experiment $1=$ A9E ZV 4.1 and passage 3 from experiment $2=$ A9E ZV 3.2. A9E lost binding to escape mutants, whereas binding was retained by G9E, 1M7, and ZKA190 as well as by all 4 primary ZIKV polyclonal plasma. A9E failed to neutralize both of the isolated escape mutants compared with potent neutralization of the wildtype (WT) positive control. However, G9E and 2 polyclonal primary ZIKV-immune plasma neutralized A9E escape mutants similarly to WT virus. Mutant viruses were sequenced and aligned to WT, with 2 mutations, one in EDIII (V364I) and the other in EDI (G128D), detected as depicted in Figure 5C.

To map the epitopes engaged by neutralizing human mAbs by a complementary approach, both A9E and G9E were epitope mapped using alanine scanning shotgun mutagenesis (Figure 5, D and E), as previously described $(45,46)$. This approach compares $\mathrm{mAb}$ binding to a library of prM/E proteins with distinct point mutations to binding of control $\mathrm{mAbs}$ that normalizes for target protein expression and folding. One critical amino acid that significantly reduced binding was detected for each mAb. For A9E, loss of binding was observed with mutation of E162, which is within EDI, proximal to the glycan at N154. This result is consistent with the A9E escape mutant containing alterations in EDI and the partial binding of this $\mathrm{mAb}$ to ZVEDI. For G9E, mutation of residue R252 resulted in loss of G9E Fab binding.

Representation of $A 9 E$ and $G 9 E$ in $Z I K V$-infected subjects. Based on escape mutations and alanine scanning mutagenesis, $\mathrm{A} 9 \mathrm{E}$ and G9E recognize distinct epitopes contained on ZIKV E. To test whether the epitopes engaged by $\mathrm{A} 9 \mathrm{E}$ and $\mathrm{G} 9 \mathrm{E}$ are frequently targeted by polyclonal plasma $\mathrm{Ab}$ in natural $\mathrm{ZIKV}$ infection and whether DENV infection could elicit cross-reactive Abs that bind similar epitopes present on ZIKV, a set of DENV- and/or ZIKV-immune plasma were competed against each mAb in BOB assays. The sources of plasma included US travelers, PCR- and serology-confirmed ZIKV cases from León, Nicaragua, and subjects from a Sri Lankan hospital-based cohort with PCR-confirmed DENV infection. The majority of DENV-immune plasma failed to block $\mathrm{mAb}$ binding to ZIKV at a level greater than $20 \%$ (Figure 6A). The samples collected from DENV-immune plasma that showed greater than $40 \%$ blockade were collected during early convalescence when cross-reactive Abs are higher (Supplemental Figure 4). Plasma specimens from ZIKV-infected individuals were further analyzed by dividing them into primary versus secondary flavivirus infection (Figure 6B) and there was no difference in the level of blockade between the 2 groups. Plasma from DT168 exhibited greater than $70 \%$ blockade for each $\mathrm{mAb}$; this was the highest level of activity among the 4 primary ZIKV-immune traveler plasma as expected, given that both mAbs were derived from DT168 peripheral blood mononuclear cells (PBMCs) (Supplemental Figure 5). Interestingly, when testing multiple specimens from the same donor 


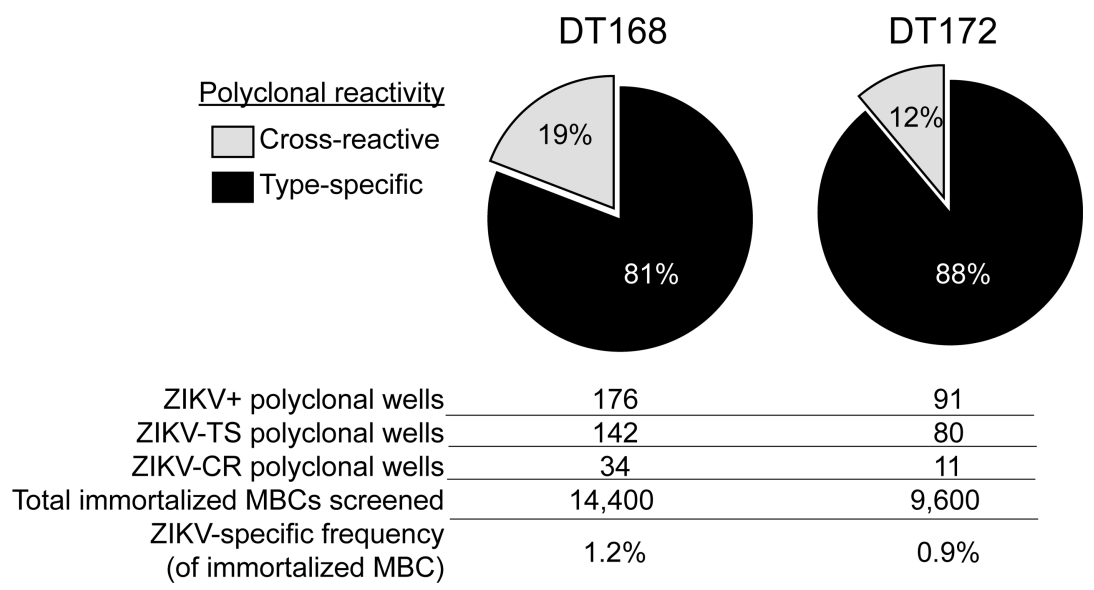

Figure 3. Frequency of ZIKV-specific and cross-reactive MBCs. MBCs were transduced using the $6 \mathrm{XL}$ method and culture supernatants assessed for ZIKV- and DENV-binding IgG. Pie charts show the proportion of ZIKV-specific and cross-reactive wells for 2 donors with prior primary ZIKV infection. The table below delineates the raw numbers used to calculate the proportions shown in pie charts and the total frequency of ZIKV-reactive MBCs for each donor. ZIKV-TS, wells were designated ZIKV-type specific when IgG ELISA result for that well was positive for ZIKV and negative for DENV antigen. ZIKV-CR, ZIKV cross-reactive, wells IgG positive for both ZIKV and DENV antigen.

at different times, the later specimen tended to have higher BOB activity. DT206 and DT244 exhibit negligible $\mathrm{BOB}$ against $\mathrm{A} 9 \mathrm{E}$ early (even through $\mathrm{FRNT}_{50}$ titers are high), but begin to show blockade ( $\left.30 \%\right)$ by 6 months after infection (Supplemental Figure 5). This suggests that BOB activity of plasma may be affected by changes in the specificities represented in the $\mathrm{Ab}$ repertoire, not just the amount of IgG being produced. To further test this hypothesis, paired samples from ZIKV cases in Nicaragua were analyzed at 21 days and 6 months after infection and the trend for 8 out of 10 specimens was an increase in BOB at the later time. Taken together, these findings indicate that, following natural ZIKV infection, $\mathrm{Ab}$ responses targeting the same antigenic region of the potent ZIKV-specific neutralizing clones we isolated are maintained into late convalescence.

\section{Discussion}

This study shows that the polyclonal Ab response in ZIKV-infected individuals comprises a complex mixture of $\mathrm{Abs}$ that recognize quaternary epitopes present on intact virions, and epitopes present on the recombinant ZIKV envelope protein monomer (simple epitopes). Furthermore, our data indicate that the majority of neutralizing activity in the 4 primary ZIKV plasma specimens is attributable to Abs that recognize quaternary epitopes. Recent studies with other flaviviruses, particularly the 4 DENV serotypes, have reached similar conclusions, suggesting that the importance of Abs targeting complex structural epitopes is a generalizable feature of the human $\mathrm{Ab}$ response to this genus of viruses (18-21, 47, 48). However, we observed in 2 of the 4 subjects that Abs targeting simple epitopes also contributed to plasma neutralizing activity (Figure 2C). Similarly, other studies have identified ZIKV-serotype-specific mAbs, which target simple epitopes on recombinant envelope proteins, particularly on EDIII, and neutralize the virus at variable potency $(34,36$, $49,50)$. Recently, our group has also found that epitopes on EDI and EDIII are frequently targeted by ZIKV-specific Abs (27). In DENV, it is known that EDIII-directed Abs generally constitute a minor component of the human neutralizing $\mathrm{Ab}$ response (51). We hypothesize that the same is likely true for ZIKV, but this is yet to be formally demonstrated. Taken together, these findings emphasize the contribution and protective role of quaternary-epitope Abs in ZIKV neutralization following primary infection.

To analyze humoral immunity in greater detail and elucidate the molecular determinants of neutralization, we examined the MBC population from 2 subjects, isolated 2 distinct potently neutralizing $\mathrm{mAbs}$ from one of the subjects, mapped their key binding determinants, and assessed the representation of these $2 \mathrm{mAb}$ specificities in a more general population. Approximately $1 \% \pm 0.04 \%$ of immortalized MBCs were $\mathrm{ZIKV}$ reactive. One caveat to this estimate is the transduction efficiency of MBCs by $6 \mathrm{XL}$, though this is accounted for by expressing frequency estimates as a function of immortalized cells. Another caveat is the possibility according to the Poisson distribution that not every well will have exactly 50 cells dispensed and cells have grown equally. Although the latter is difficult to assess, we have found up to a $4 \%$ variance in 
Table 3. Derivation of polyclonal and monoclonal memory B cells from DT168

\begin{tabular}{|c|c|c|c|c|c|c|c|c|c|c|c|}
\hline \multicolumn{12}{|c|}{ Subject DT168 } \\
\hline Polyclonal & & $\rightarrow$ & \multicolumn{8}{|c|}{ Monoclonal Cultures } & \multirow{2}{*}{\begin{tabular}{|c} 
Notes \\
IGH/IGL \\
sequencing
\end{tabular}} \\
\hline Well ID & $\mathrm{OD}(\mathrm{ZIKV})^{\mathrm{A}}$ & & $\begin{array}{c}\text { No. cells } \\
\text { sorted }\end{array}$ & $\begin{array}{l}\text { No. viable } \\
\text { cultures }\end{array}$ & Recovery & $\begin{array}{l}\text { No. ZIKV }{ }^{+} \\
\text {wells }\end{array}$ & $\begin{array}{c}\text { OD (ZIKV) } \\
\text { of positives }^{A} \\
\text { (range) }\end{array}$ & $\begin{array}{l}\text { Unique } \\
\text { clones }^{B}\end{array}$ & $\begin{array}{l}\text { Frequency } \\
\text { unique } \\
\text { positive } \\
\text { clones in } \\
\text { polyclonal }\end{array}$ & $\begin{array}{c}\text { No. ZIKV } \\
\text { neutralizers } \\
\text { (activity) }^{\complement}\end{array}$ & \\
\hline E4 & 0.54 & $\rightarrow$ & 180 & 89 & $49 \%$ & 68 & $\begin{array}{l}0.23 \pm 0.03 \\
(0.20-0.30)\end{array}$ & 2 & $4 \%(2 / 50)$ & $1(51 \%)$ & N.D. \\
\hline F11 & 0.51 & $\rightarrow$ & 120 & 20 & $17 \%$ & 0 & - & - & - & - & N.D. \\
\hline $\mathrm{H} 10$ & 0.49 & $\rightarrow$ & 120 & 53 & $44 \%$ & 5 & $\begin{array}{l}0.25 \pm 0.01 \\
(0.25-0.27)\end{array}$ & 1 & $2 \%(1 / 50)$ & $1(58 \%)$ & N.D. \\
\hline B3 & 0.46 & $\rightarrow$ & 120 & 18 & $15 \%$ & 0 & - & - & - & - & N.D. \\
\hline D10 & 0.44 & $\rightarrow$ & 120 & 60 & $50 \%$ & 0 & - & - & - & - & N.D. \\
\hline C3 & 0.42 & $\rightarrow$ & 120 & 52 & $43 \%$ & 0 & - & - & - & - & N.D. \\
\hline G11 & 0.42 & $\rightarrow$ & 120 & 53 & $44 \%$ & 9 & $\begin{array}{l}0.19 \pm 0.02 \\
(0.14-0.22)\end{array}$ & 1 & $2 \%(1 / 50)$ & $1(58 \%)$ & N.D. \\
\hline F9 & 0.42 & $\rightarrow$ & 120 & 26 & $22 \%$ & 0 & - & - & - & - & N.D. \\
\hline $\begin{array}{l}\text { Mean } \pm \text { SD } \\
\text { or Totals }\end{array}$ & $0.48 \pm 0.06$ & & 1260 & 460 & $37 \%$ & 101 & $0.27 \pm 0.06$ & 7 & $\begin{array}{c}2.8 \% \\
(1.4 / 50)\end{array}$ & 6 & \\
\hline
\end{tabular}

${ }^{A}$ Mean $\pm S D$ of $O D$ for ZIKV-binding ELISA (background $=0.1-0.15$ ). ${ }^{B}$ Equals 1 if $S D \leq 10 \%$ of mean, equals 2 if $S D>10 \%$ of mean, confirmed by sequencing of $\mathrm{D} 1$ and $\mathrm{E3}$ clones. ${ }^{\mathrm{C}}$ Number positive is defined as $\geq 50 \%$ neutralization of ZIKV; percentage neutralization is presented in parentheses. ${ }^{\mathrm{C}} \mathrm{Clone} \mathrm{C} 9 \mathrm{E}$ with $83 \%$ neutralization was sequenced. "A9E" full name is DT168 (A)-E3-(A)-E9. "C9E" full name is DT168 (A)-D1-(G)-E9. N.D., not determined.

observed versus expected cell dispensing after sorting. Given these limitations, our frequency estimates are within the expected range for antigen-specific MBC responses to DENV (52) and ZIKV (53), suggesting adequate sampling of the MBC pool. The presence of replacement mutations in the FWRs and CDRs of $I G V H / L$ genes of MBC clones from which A9E and G9E were derived suggested that these MBCs have undergone somatic mutation. In these clones, the CDRs contained more replacement mutations than the FWRs. This is consistent with the low tolerability of FWR replacement mutations compared with CDR replacement mutations (54). This is because FWRs are essential to maintain variable-region structural integrity $(55,56)$ and positive selection of clones $(57)$. Interestingly, A9E exhibited a positive ratio of replacement to neutral FWR mutations in IGHV akin to potent anti-HIV mAbs (58). Functionally, we found the vast majority of antigen-specific MBC clones isolated from primary ZIKV cases to be ZIKV specific and not cross-reactive to DENV. Others have clearly shown that ZIKV infection in a DENV-immune host activates preexisting, cross-reactive MBC responses (34-36), which means the repertoire selected when ZIKV is a primary versus secondary flavivirus infection could be distinct and have consequences for virus control, clinical outcome, and transmission. Our results also raise the question of how the minor population of DENV cross-reactive MBCs generated following primary ZIKV infection will contribute to the humoral response to subsequent DENV infection.

Identifying targets of the long-lived neutralizing $\mathrm{Ab}$ response is a fundamental requirement for vaccine development, as these may guide further antigen design as well as assessment of vaccine-induced immunity. For DENV $(18-20,47)$ and other flaviviruses $(21,48)$, it has been observed that strongly neutralizing, type-specific Abs often target quaternary epitopes, and our data support the same conclusion for primary ZIKV-immune individuals (Figure 2D). Interestingly, the 2 potently neutralizing $\mathrm{mAbs}$ isolated in our study bound to recombinant ZIKV envelope protein monomer. Depletion experiments (Figure 2) are 
consistent with subject DT168 having a neutralizing Ab response against ZIKV that recognizes both simple and complex structural epitopes. An expanded set of mAbs from DT168 and other ZIKV cases is being isolated for comparative study. It is highly likely that mAbs against quaternary ZIKV epitopes will be isolated from DT168. Additionally, binding of the ZIKV $\mathrm{E}$ monomer does not preclude that residues on adjacent $\mathrm{E}$ proteins be included in the $\mathrm{mAb}$ footprint nor that these potential residues be critical for the mechanism of neutralization by A9E and G9E. Thus, binding experiments such as those performed here are an important part of $\mathrm{mAb}$ characterization but are limited in that structure and function cannot be inferred from results. Therefore, additional work is needed to fully understand the properties of these $2 \mathrm{mAbs}$ as well as the primary $\mathrm{Ab}$ response to $\mathrm{ZIKV}$ in general.

We found that $\mathrm{A} 9 \mathrm{E}$ and $\mathrm{G} 9 \mathrm{E}$ recognize distinct epitopes based on lack of competitive binding by each other and on different critical binding residues identified by complementary epitope mapping approaches. A9E binding was blocked by ZKA190, whose epitope spans the lateral ridge of EDIII and residues in the EDI/EDIII linker region (43). EDI likely contains part of, but not the entire, A9E footprint based on ZKA190 competition and the weaker binding of EDI versus ZIKV E80 exhibited by A9E. It was not possible to generate an escape mutant to G9E, perhaps because the footprint of G9E includes at least one critical residue essential for viral fitness. G9E appears to bind residues primarily in EDII because mutagenesis revealed loss of binding with R252A, and this mAb did not bind monomeric EDI or EDIII. Moreover, BOB by EDE1 Abs (C8 and C10) supports an epitope in EDII. Taken together, our data suggest that the epitopes of these $2 \mathrm{Abs}$ do not overlap. In fact, we have solved a high-resolution crystal structure for G9E and observed that the footprint of this mAb spans the homodimer (Adams, unpublished observation).

Antigen-specific responses arise under the influence of a variety of host- and pathogen-specific features, which leads to certain responses being particular to an individual (private), while others are more broadly represented in populations (public). The latter would need to be true for tracking of an antigen-specific response to be a useful tool for vaccine development. In general, plasma Abs from ZIKV-immune individuals (including those with and without prior DENV infection) competed with A9E and G9E for ZIKV virion binding. DENV-immune plasma seldom blocked binding of A9E and G9E to ZIKV, or it did so with substantially less efficiency. Interestingly, ZIKV-immune plasma from later times ( $>1$ month and typically 6 months after infection) exhibited a greater degree of blocking activity. Overall neutralization titers typically peak and decline before 6 months, which suggests that this effect is not simply due to total amount of IgG present in the plasma, but may involve ongoing shaping of specificities maintained in the $\mathrm{Ab}$ repertoire for months following acute infection. Although these results do not prove that the exact epitope of either mAb is widely targeted in people with ZIKV infection, they do indicate that the region of E surrounding the A9E and G9E epitopes appears to be highly immunogenic in human ZIKV infection.

It is important to note that in vitro selection of escape mutants is a tool for epitope mapping and does not indicate that these same escape mutants are likely to arise in nature. The experimental conditions used in the lab do not model natural infection; the immunologic pressure exerted by a naturally infected host is unlikely to be entirely against a single epitope, a point recently illustrated by a ZIKV glycosylation mutant that confers escape to a specific $\mathrm{mAb}$ but not to polyclonal plasma (59). Additionally, flaviviruses are subject to purifying selection due to both mammalian and insect hosts in their life cycle (60).

The primary goal of this work was to study the primary Ab response to ZIKV in detail, but we identified 2 ZIKV mAbs with potential for further development for therapeutic (43, 46) or diagnostic (61) purposes. The $\mathrm{FRNT}_{50}$ values of A9E (3-17 ng/ml) and G9E (20-38 $\mathrm{ng} / \mathrm{ml}$ ) are among the most potent reported for native human ZIKV mAbs. Multiple strains of ZIKV, representing African and Asian lineages, were effectively neutralized, consistent with the idea that ZIKV exists as a single serotype $(62,63)$. Additionally, A9E and G9E both fail to bind or neutralize DENV, and both protected against murine lethal ZIKV challenge in vivo. Finally, these $2 \mathrm{mAbs}$ appear to define epitopes that are consistently targets of the $\mathrm{Ab}$ response to natural ZIKV infection, as evidenced by the BOB studies with our initial set of 
A

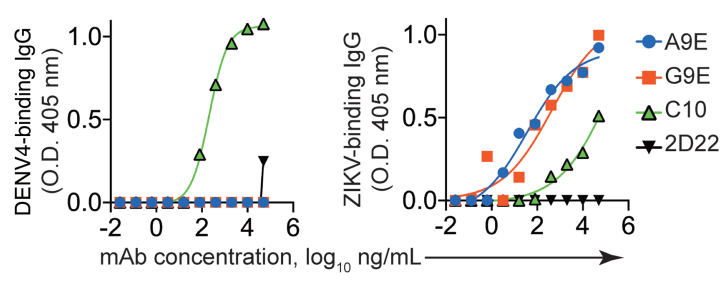

C

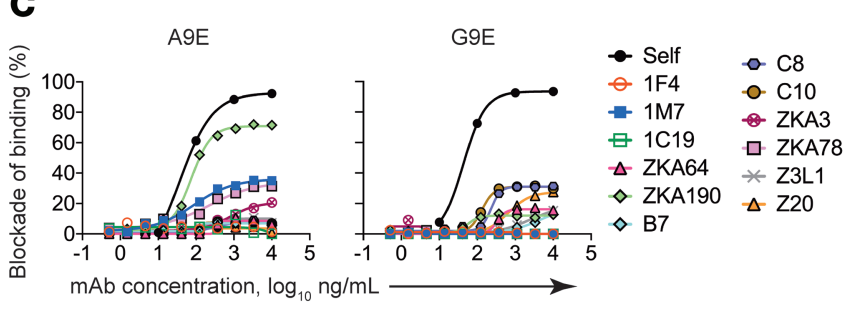

$\mathbf{F}$

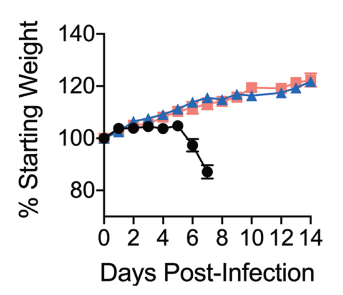

G

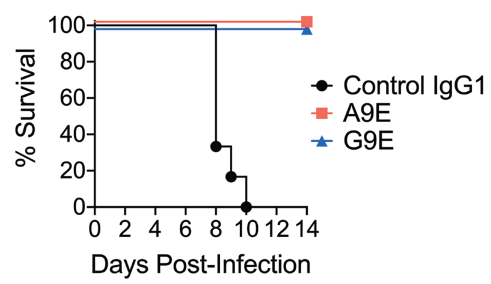

B
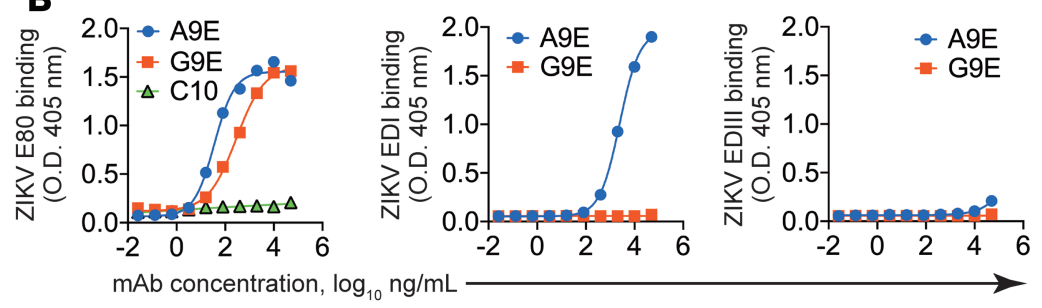

$\mathbf{E}$

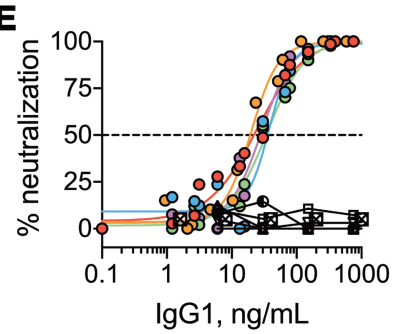

Figure 4. mAbs from primary ZIKV case exhibit potent ZIKV-specific neutralization and confer in vivo protection against lethal ZIKV challenge. (A) Antigen-capture ELISA for IgC binding was performed for 2 candidate ZIKV mAbs and 2 control mAbs (C10, ZIKV and DENV neutralizing; 2D22, DENV2 neutralizing) against DENV4 (left) and ZIKV (right). (B) Binding to ZIKV E monomers and EDI and EDIII was assessed for each mAb. (C) Competition assays (BOB) with a panel of mAbs with known binding specificities was performed to localize the epitopes of A9E and G9E. (D and E) FRNT assays against a panel of ZIKV strains and related flaviviruses were performed for A9E (D) and G9E (E). (F and G) Four- to 6-week-old Ifnar ${ }^{-/-}$mice were treated with 200 $\mu \mathrm{g}$ of indicated A9E, G9E, or polyclonal human IgG as a negative control on day -1 and challenged with 1,000 FFU of ZIKV (H/PF/2013). Weight loss (F) and mortality (G) were monitored for 14 days after infection. Results represent 6 to 7 mice per group combined from 2 independent experiments. Weights are shown as mean \pm SEM and were censored upon the first death in the group. NA, not applicable.

human plasma from ZIKV-infected individuals. Additional experiments, including more detailed in vivo work to assess various timing and dose schedules and for breakthrough or persistent viremia, are being pursued to further explore the prophylactic and therapeutic potential of the mAbs.

Because people exposed to primary DENV infections also develop Abs cross-reactive to heterologous DENV serotypes with the potential to enhance viral replication and disease during a secondary heterotypic DENV infection (64-66), potential enhancement of ZIKV disease by cross-reactive Abs elicited by prior DENV infection was a major concern early in the recent ZIKV epidemic. However, the grounds for this hypothesis is limited to in vitro experiments $(33,36,67-69)$ and mouse models (70), but has not borne out in nonhuman primate studies $(31,71)$ or epidemiologically in humans $(30,72)$. It is also plausible that ZIKV infection may elicit cross-reactive Abs that could later enhance a subsequent DENV infection $(32,36,73)$. The mAbs we have identified here do not bind DENV, precluding the possibility of causing Ab-dependent enhancement (ADE) of DENV infection. Enhancement of $\mathrm{ZIKV}$ infection is a theoretical possibility, but very unlikely as there is no evidence of ADE occurring in natural ZIKV infection of humans. Additionally, in contrast to DENV, all ZIKV strains constitute a single serotype (62), and we have shown that A9E and G9E potently neutralize different ZIKV strains from the Asian and African lineages. We further show that A9E- and G9E-like ZIKV-specific binding is present in a substantial portion of primary ZIKV cases and such activity increases over time. This suggests a narrowing of the repertoire towards ZIKV specificity typified by A9E- and G9E-like reactivity, indicating limited potential for DENV enhancement by Abs of this type. 
A
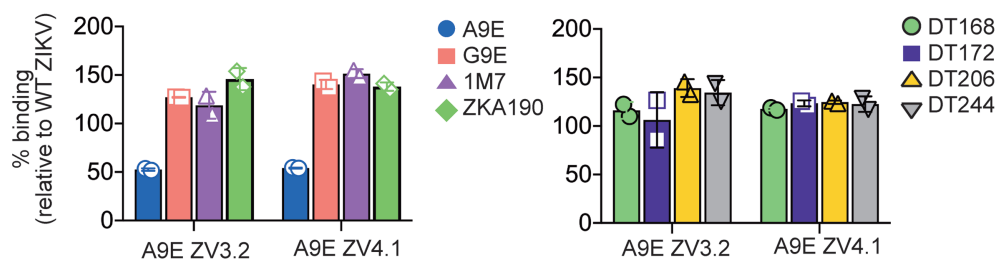

C

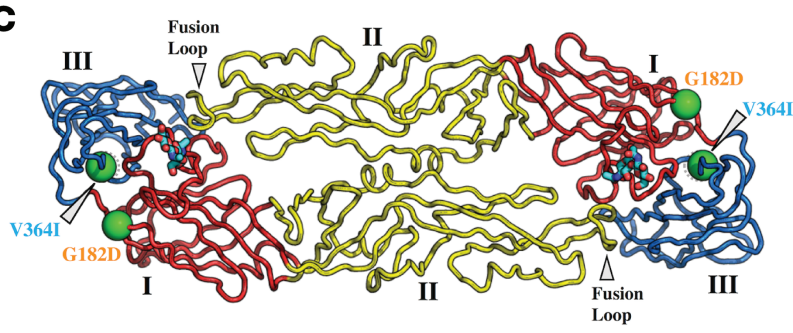

D

A9E

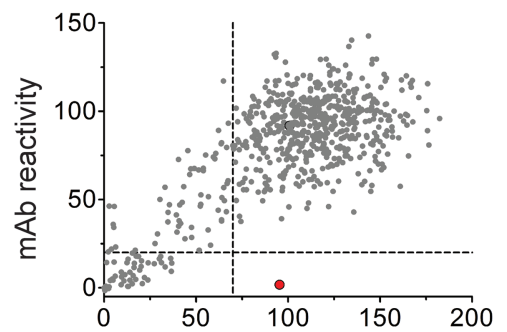

Control mAb reactivity
B

A9E
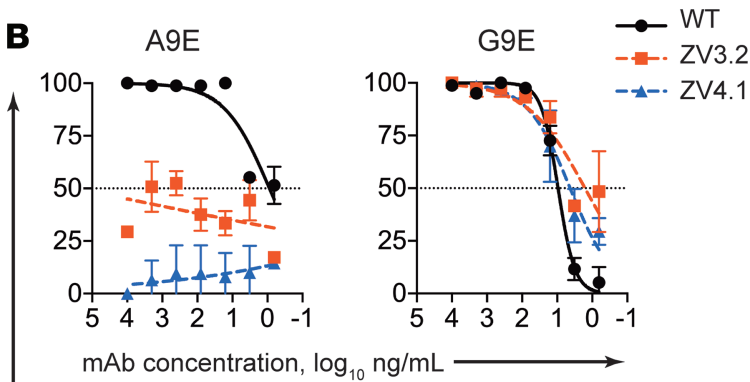

$\mathrm{mAb}$ concentration, $\log _{10} \mathrm{ng} / \mathrm{mL}$

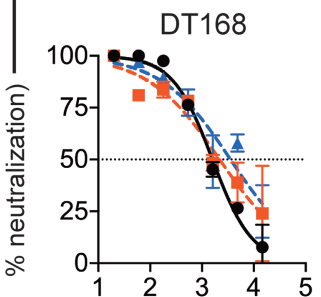

$\log _{10}[1 /$ serum dilution $]$

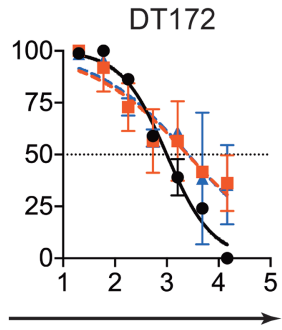

E A9E

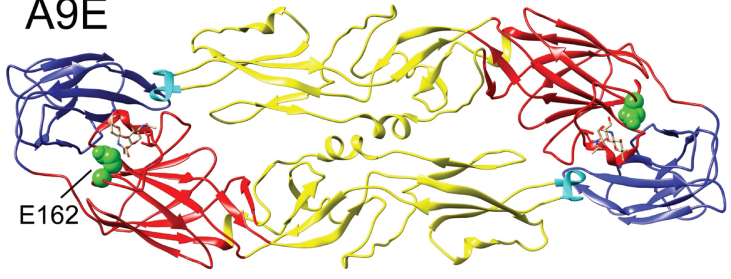

F

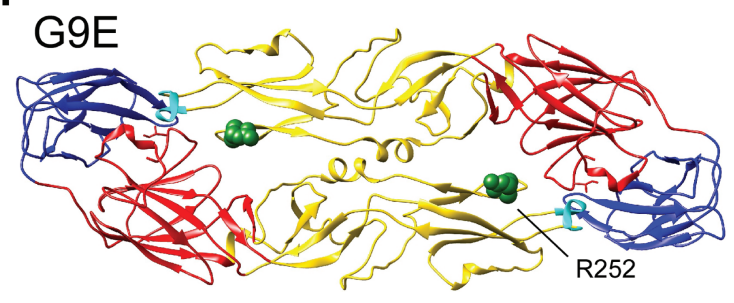

Figure 5. Epitope mapping of ZIKV-neutralizing mAb. (A-C) Escape mutants for A9E were generated from PRVABC59. (A) Binding of indicated mAb (left) and plasma (right) against A9E escape mutants from 2 independent experiments is shown. (B) Neutralization of 2 A9E escape mutants from 2 independent experiments by indicated mAb (top) and plasma (bottom) is shown. (C) ZIKV E homodimer with escape mutations indicated. (D) Amino acid residues critical for A9E mAb and G9E Fab binding were determined by alanine scanning shotgun mutagenesis. Plots show the binding of A9E and G9E versus control mAbs. The data point in red corresponds to the alanine mutant that significantly reduces probe mAb binding compared with loading control mAbs. (E) Critical residues (green spheres) discovered in alanine mutagenesis mapping are represented on a 3-dimensional model from a ZIKV cryo-EM structure (PDB ID: 5IRE). The fusion loop of E domain II is in cyan, domain I is in red, domain II is in yellow, and domain III is in blue.

In conclusion, this work provides a foundation for further definition of the molecular determinants of ZIKV neutralization and the durable humoral responses that will contribute to protection against infection. The tools and approaches used here will be useful in assessing $\mathrm{Ab}$ and $\mathrm{MBC}$ responses elicited by candidate ZIKV vaccines and the further study of $\mathrm{Ab}$ responses elicited by sequential, heterologous flavivirus infection.

\section{Methods}

Human subjects and biospecimen collection

UNC travelers. Plasma was collected from North Carolina residents with self-reported risk for or diagnosis of arbovirus infection through travel. Plasma samples were tested by virus-capture ELISA. DENV- or ZIKV-reactive plasma was further characterized by neutralization assays on Vero cells to verify prior flavivirus infection. 

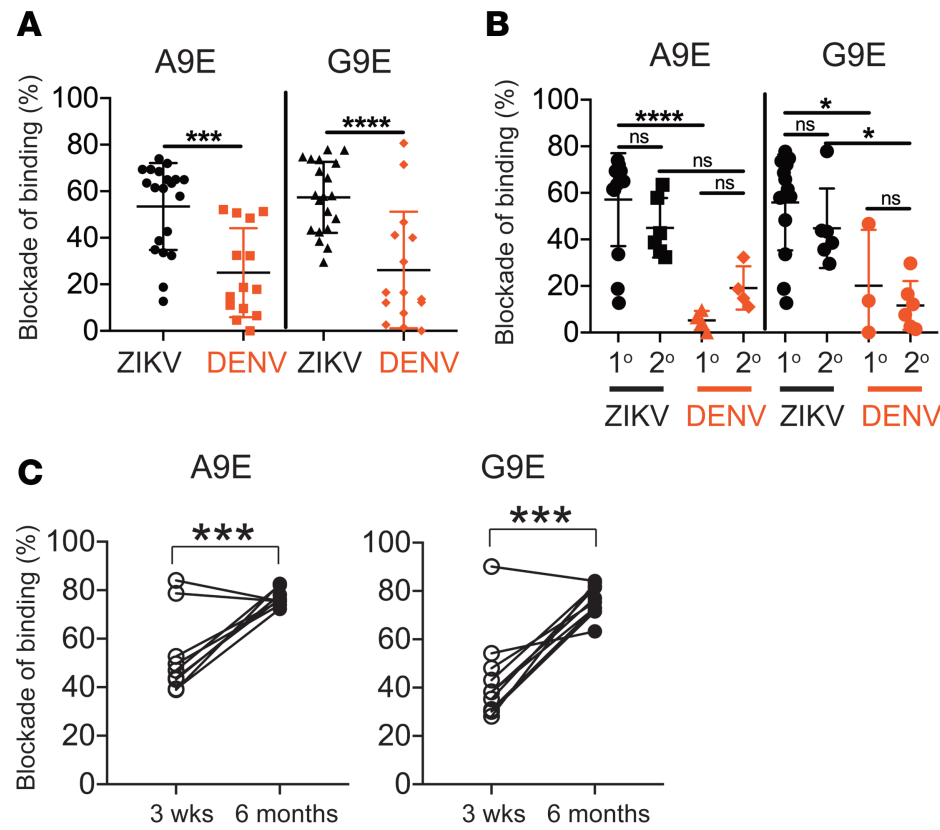

Figure 6. A9E and G9E epitope-binding IgGs are widely represented in polyclonal plasma following natural ZIKV infection. (A) Blockade of binding (BOB) against A9E and G9E was tested among plasma at a 1:20 dilution from ZIKV and DENV cases from the UNC Traveler's study, Nicaragua, and Sri Lanka, as was done for mAbs in Figure 4C. (B) The ZIKV cases were subdivided into primary $\left(1^{\circ}\right)$ and secondary $\left(2^{\circ}\right)$ ZIKV (ZIKV infection in a DENV-immune host). (C) Paired plasma specimens at 1:10 dilution from symptomatic ZIKV cases in Nicaragua were analyzed by BOB at early (day 21 after symptom onset) versus late (6 months after symptom onset) convalescence. An unpaired Student's $t$ test was performed to determine differences in means between groups as indicated by bars. ${ }^{* *} P<0.01 ;{ }^{* *} P<0.001$; ${ }^{* * * *} P<0.0001$. ns, not significant.

Plasma that neutralized one DENV serotype or ZIKV with minimal neutralizing activity against other viruses were defined as primary flavivirus infections (meaning that the $\mathrm{FRNT}_{50}$ for a single DENV serotype or ZIKV is at least 4-fold higher than any other virus tested). In our ZIKV cases, the travel history of the subject corroborated the primary ZIKV immune status. Secondary flavivirus infections were defined by the highest 2 or more $\mathrm{FRNT}_{50}$ values with less than 4-fold separation. Existing plasma with known flavivirus neutralization profiles were used as controls in several experiments: primary $\left(1^{\circ}\right)$ DENV neutralized a single DENV serotype and not ZIKV; secondary $\left(2^{\circ}\right)$ DENV neutralized at least 2 DENV serotypes and not ZIKV.

Nicaraguan subjects. Patients presenting with fever, rash, or nonsuppurative conjunctivitis in León, Nicaragua, were recruited to a prospective cohort study, in which ZIKV cases were identified by reverse transcription PCR (RT-PCR) on site and confirmed serologically at the University of North Carolina (UNC). ZIKV cases were sampled by blood draw at presentation and at weeks 2, 3, 4, 8, 12, and 24 after symptom onset.

Sri Lankan subjects. During a DENV1 epidemic in Sri Lanka in 2014, suspected symptomatic DENV cases were enrolled for prospective sampling. Cases were confirmed by RT-PCR. All subjects were enrolled within 4 days of symptom onset and a convalescent blood sample was obtained (ranging from 16-29 days after onset of symptoms).

Viruses and cells

The MR766 and Dakar 41525 strains of ZIKV were obtained from the World Reference Center for Emerging Viruses and Arboviruses (R. Tesh, University of Texas Medical Branch, Galveston, Texas, USA) (74, 75). ZIKV strains $\mathrm{H} / \mathrm{PF} / 2013$ and PRVABC59 were provided by the US Centers for Disease Control and Prevention (76, 77). ZIKV/2012/PHL (Genbank: KU681082), ZIKV/2014/TH (Genbank: KU681081.3), and ZIKV/2015/Paraiba (Genbank: KX280026.1, PMID 27555311) were obtained from Stephen Whitehead (National Institute of Allergy and Infectious Diseases, NIH). DENV WHO reference strains DENV1 West Pac 74, DENV2 S-16803, DENV3 CH54389, and DENV4 TVP-360 were initially obtained from Robert Putnak (Walter Reed Army Institute of Research, Silver Spring, Maryland, USA). DENV2 NGC, DENV2/1974/Tonga (Genbank: AY744147.1), DENV3/1978/Slemen (Genbank: AY648961.1), and 
DENV4/1981/Dominica (Genbank: AF326573.1) were used in neutralization experiments and were obtained from Stephen Whitehead. To perform culture-based experiments and maintain virus stocks, C6/36 Aedes albopictus cells (ATCC CRL-1660) or Vero (Cercopithecus aethiops) cells (ATCC CCL-81) were used. $\mathrm{C} 6 / 36$ cells were grown at $32^{\circ} \mathrm{C}$ with $5 \% \mathrm{CO}_{2}$ in $\mathrm{MEM}$ supplemented with $10 \%$ fetal bovine plasma, L-glutamine, nonessential amino acids, and HEPES buffer. Vero cells were grown at $37^{\circ} \mathrm{C}$ with $5 \% \mathrm{CO}_{2}$ in DMEM supplemented with 5\% fetal bovine plasma and L-glutamine. Virus stocks were titrated on Vero cells by plaque assay or focus-forming assay. All studies were conducted under biosafety level 2 containment.

\section{Human mAb generation and identification}

From subject DT168, mAbs were generated as previously described using the 6XL method (39). Briefly, total cryopreserved PBMCs were thawed and MBCs isolated by magnetic purification for $\mathrm{CD} 22^{+} \mathrm{B}$ cells and flow cytometric sorting for $\mathrm{CD} 19^{+} \mathrm{CD} 27^{+} \mathrm{IgM}^{-}$class-switched MBCs. MBCs were transduced with $6 \mathrm{XL}$ retrovirus (encodes both Bcl-6 and Bcl-xL) and activated with CD40L-expressing cells and recombinant human interleukin 21 (IL-21), which together support proliferation and secretion of Ab (78). Transduced cells were sorted by GFP expression (transduction marker) into polyclonal cultures at $50 \mathrm{GFP}^{+}$cells/well on 96-well plates using fluorescence-activated cell sorting on a BD FACSAria III using the "single cell sort mask" mode, which provides the highest possible accuracy ( $<4 \%$ variance) in dispensing cells. Supernatants from polyclonal cultures were tested for the presence of IgG targeting ZIKV by capture ELISA. ZIKV-specific supernatants were further screened for cross-reactivity to DENV in capture ELISA, and for ZIKV E80 binding in direct antigen-coating ELISA. Selected ZIKV-specific polyclonal cultures were single-cell sorted into monoclonal cultures on a BD FACSAria III, grown on CD40L and IL-21, and then screened as above after 4 weeks. ZIKV-specific monoclonal cultures were further qualitatively tested for neutralization of ZIKV by incubation of ZIKV with $30 \mu \mathrm{l}$ of culture supernatants prior to infection of Vero cells and assessment of neutralizing activity by microneutralization assay (Supplemental Figure 3).

From frozen cell pellets of monoclonal cultures we isolated RNA, performed nested PCR for IGH and $I G L$ genes, and sequenced using specific primers as described previously $(40,41)$. Sequences were input into IgBLAST (https://www.ncbi.nlm.nih.gov/igblast/) and compared to germline to determine variable heavy and light chain usage, V-(D)-J gene usage, SHMs, CDR3 sequence, and IgG subtype. Because sequencing of both of the potently neutralizing mAbs revealed IgG1 isotype and Ig- $\lambda$ light chain usage, we then used previously described methods $(40,41)$ to clone IGH and IGL into human IgG1 (Genbank, FJ475055) and Ig $\lambda$ expression vectors (Genbank, FJ517647), respectively. Heavy and light chain vectors were verified by sequencing and cotransfected into HEK-293F cells and mAbs were produced as described previously $(40,41)$.

\section{ELISA}

Binding of mAb or plasma IgG to DENV or ZIKV was measured by capture ELISA as described previously (20). Briefly, DENV or ZIKV virions were captured by the anti-E protein mouse mAb 4G2, blocked with 3\% nonfat dry milk (LabScientific, Inc), and incubated with mAb or human plasma at indicated dilutions at $37^{\circ} \mathrm{C}$ for 1 hour, and binding was detected with an alkaline phosphatase-conjugated goat anti-human IgG secondary Ab (MilliporeSigma) and p-nitrophenyl phosphate substrate (MilliporeSigma). Absorbance at $405 \mathrm{~nm}$ (OD) was measured on an Epoch or Cytation3 plate reader system (Bio-Tek). ELISA assays to measure recombinant antigen binding (ZIKV E80, ZVEDI, and ZVEDIII) or used to confirm depletion were performed as above with the exception that $50 \mathrm{ng}$ purified antigen was coated directly on the plate at $37^{\circ} \mathrm{C}$ for 1 hour.

ELISA data are reported as OD values that are the average of technical replicates unless otherwise indicated. Naive human plasma (NHS) served as the negative control in ELISA assays. In depletion experiments, the OD of depleted sample is expressed as percentage of control from the same plasma as indicated. For IgG binding to myelin basic protein-tagged (MBP-tagged) fusion proteins ZVEDI and ZVEDIII, the OD values reported are background subtracted for each plasma individually (OD ZIKV - OD MBP).

\section{BOB assay}

BOB was performed as described previously (79). Briefly, ZIKV was captured using 4G2 and plates were blocked with $3 \%$ nonfat dry milk as for ELISA. Serial dilutions of plasma were added to plates in duplicate and incubated at $37^{\circ} \mathrm{C}$ for 1 hour. After washing, $100 \mathrm{ng} /$ well of alkaline phosphatase-conjugated 
G9E or A9E was added, and plates were incubated at $37^{\circ} \mathrm{C}$ for 1 hour. $p$-Nitrophenyl phosphate substrate was added, and color changes were quantified by spectrophotometry. Percentages of blockade of binding were calculated as follows: $(100-$ [OD of sample/optical density of control] $) \times 100$.

\section{Neutralization assays}

Neutralization titers were determined by 96 -well microFRNT $(38,80)$. Serial dilutions of mAb or plasma were mixed with approximately 50-100 FFUs of virus in DMEM with $2 \%$ FBS. The virus-Ab mixtures were incubated for 1 hour at $37^{\circ} \mathrm{C}$ and then transferred to a monolayer of Vero cells for infection for 2 hours at $37^{\circ} \mathrm{C}$. OptiMEM overlay media (Gibco, 31985) supplemented with $2 \% \mathrm{FBS}$, $1 \%$ Anti-Anti, and $5 \mathrm{~g}(1 \%$ ) carboxymethylcellulose (MilliporeSigma, C-5013) was added, and cultures were incubated for 40 hours (ZIKV), 48 hours (DENV2 and DENV4), or 52 hours (DENV1, DENV3). Cells were fixed with $70 \mu \mathrm{l}$ of $4 \%$ paraformaldehyde (Thermo Fisher Scientific, 28908) for 30 minutes. Permeabilization buffer $(100 \mu \mathrm{l})$ was added for 10 minutes followed by $100 \mu \mathrm{l}$ of blocking buffer $(3 \%$ normal goat plasma, MilliporeSigma G-9023 in permeabilization buffer) and left overnight at $4^{\circ} \mathrm{C}$. Fifty microliters of a mixture of primary Abs 4G2 and 2H2 (81) (ATCC, HB-114; $2 \mathrm{H} 2$ not used for ZIKV) was added to the plates and incubated for a 1 hour at $37^{\circ} \mathrm{C}$. Cells were washed with a microplate washer (Bio-Tek, ELx405) followed by the addition of $50 \mu$ of 1:1900 horseradish peroxidase-conjugated goat anti-mouse secondary $\mathrm{Ab}(\mathrm{KPL}, 074-1806)$ for 1 hour at $37^{\circ} \mathrm{C}$. Foci were visualized with $60 \mu 1$ of True Blue (KPL, 5510-0030) and counted with a user-supervised automated counting program on $\times 2$-magnified images of microwells obtained on a CTL ELISPOT reader. Two NHS controls on each plate defined $100 \%$ infection.

\section{Ab depletions}

$\mathrm{ZIKV}$ recombinant E protein was purified as described previously (82) and conjugated to HisPur Ni-NTA magnetic beads (Thermo Fisher Scientific) per manufacturer's instructions. Control beads were incubated with an equal amount His-tagged human MBP (His-MBP). For depletion, plasma were diluted 1:20 and incubated with $30 \mu \mathrm{g}$ ZIKV E80 or His-MBP control split over 2 rounds for 1 hour at $37^{\circ} \mathrm{C}$ per round. Depletion was confirmed by a ZIKV E80-binding ELISA. Plasma were also depleted of all ZIKV binding Abs using ZIKV VLPs as previously described (83). ZIKV VLPs were provided by The Native Antigen Company and produced by transiently expressing ZIKV prM and E proteins in suspension culture-adapted HEK-293 cells. Supernatants were cleared by centrifugation and concentrated by tangential flow filtration. The VLPs were purified by discontinuous sucrose gradient, ion exchange chromatography, and size exclusion chromatography, which also provided exchange of buffers to storage buffer. Purified VLPs were stored in $10 \mathrm{mM}$ sodium phosphate, $20 \mathrm{mM}$ sodium citrate, $154 \mathrm{mM}$ sodium chloride, $\mathrm{pH} 7.4$ at $-80^{\circ} \mathrm{C}$ until further use.

\section{Escape mutant selection and sequence analysis}

ZIKV-PRVABC59 was incubated for 1 hour at $37^{\circ} \mathrm{C}$ with various concentrations of mAb at 2 times the $\mathrm{FRNT}_{50}$ for initial escape selection. The mAb concentration was increased every 3-6 passages up to a maximum concentration of $1,000 \times \mathrm{FRNT}_{50}$. Vero cell monolayers in 6-well tissue culture plates were infected with ZIKV-mAb mixture at a MOI of 0.01 for 2 hours at $37^{\circ} \mathrm{C}$. Vero cells were washed 3 times with PBS, and media with the same concentration of selecting mAb were replaced. Cultures were incubated up to 96 hours and checked daily for cytopathic effects. Virus growth in the presence of $\mathrm{Ab}$ was monitored by quantitative RT-PCR and by immunofluorescent detection of ZIKV antigens in cell monolayers. WT ZIKV-PRVABC59 was passaged in media alone alongside virus undergoing $\mathrm{mAb}$ selection. The E gene of stock, WT passaged, and escape mutants were sequenced and aligned in Vector NTI. Mutations resulting in changes in predicted amino acids were visualized in topographical models using PyMOL.

\section{Epitope mapping}

Alanine scanning mutagenesis was carried out by Integral Molecular on an expression construct for ZIKV prM/E (strain ZikaSPH2015; UniProt accession Q05320). Residues were mutagenized to create a library of clones, each with an individual point mutant (46). Residues were changed to alanine (with alanine residues changed to serine). The resulting ZIKV prM/E alanine-scanlibrary covered $100 \%$ of target residues(672 of 672). 
Each mutation was confirmed by DNA sequencing, and clones were arrayed into 384-well plates, one mutant per well. Cells expressing each ZIKV E mutant were immunostained with the mAb to be mapped and control $\mathrm{mAbs}$ to normalize for protein expression levels. Mean cellular fluorescence was detected using an Intellicyt flow cytometer. If no critical mutations were identified in the initial screen, $\mathrm{mAb}$ was converted to $\mathrm{Fab}$ and rescreened. Mutations within critical clones were identified as critical to the $\mathrm{mAb}$ epitope if they did not support reactivity of the mAb, but did support reactivity of conformation-dependent control mAbs. This counter-screen strategy facilitates the exclusion of Env mutants that are globally or locally misfolded or that have an expression defect (45). Validated critical residues represent amino acids whose side chains make the highest energetic contributions to the mAb-epitope interaction $(84,85)$.

\section{Mouse protection experiments}

Five-week-old male and female Ifnar1 ${ }^{-1-}$ mice (C57BL/6 background) received $200 \mu \mathrm{g}$ of A9E, G9E, or IgG1 isotype control by intraperitoneal injection 1 day prior to infection with 1,000 FFU of ZIKV (H/PF/2013) by subcutaneous footpad inoculation (44). Weight and lethality were monitored daily for 14 days.

\section{Statistics}

FRNT $_{50}$ values were determined in neutralization assays by using the sigmoidal dose response (variable slope) equation of Prism 6 (GraphPad Software). Maximum (100\%) infection was defined by the foci counts in negative control wells (negative plasma or non-neutralizing $\mathrm{mAb}$ ). $\mathrm{FRNT}_{50}$ values are the calculated titer at which maximum foci counts are reduced by $50 \%$. Dilution curves for plasma $\mathrm{Ab}$ and $\mathrm{mAb}$ binding were generated using the same equation. Reported $\mathrm{FRNT}_{50}$ values were required to have an $R^{2}$ greater than 0.75 , a Hill slope greater than 0.5 , and an $\mathrm{FRNT}_{50}$ falling within the range of the dilution series. Kaplan-Meier curves were used to establish survival differences in mouse challenge experiments. An unpaired, 2-tailed Student's $t$ test was performed to compare between groups of plasma tested in BOB experiments.

\section{Study approval}

All donations at UNC at Chapel Hill were collected in compliance with the UNC Institutional Review Board (IRB) (protocol 08-0895). Informed consent/assent or parental consent was obtained for all Nicaraguan subjects under approval of the Ethics Committee of the Universidad Nacional Autónoma de Nicaragua-León (Acta 37, 2017) and UNC IRB (protocol 16-0541). The hospital-based study in Sri Lanka was approved by the Ethical Review Committee of the Faculty of Medicine, University of Colombo, Sri Lanka, and de-identified specimens were shared with UNC for further analysis. All mouse experiments were conducted under the approval of the UNC IACUC, in AAALAC-accredited facilities.

\section{Author contributions}

MHC wrote the manuscript with input from the other authors. MHC, HAT, CGO, AMDS, and SAD designed the study and interpreted findings. HAT generated transformed polyclonal and monoclonal MBC libraries. HML performed in vivo challenge experiments, provided ZIKV reagents, and edited the manuscript. CGO generated and tested escape mutants. HAT, RSJ, GJAL, BDM, SWM, AT, and CGO performed experiments and analyzed data. BJD and ED performed epitope mapping. FB, NMB, SBD, and YR led the Nicaraguan cohort study and provided clinical specimens.

\section{Acknowledgments}

We thank the study volunteers and clinical staff at the UNC Travelers Clinic and Edwin Centeno for assistance with specimens from León, Nicaragua. This study was supported by NIH grants R01AI107731 (to AMDS), 5T32AI007151-40 (to MHC), T32AI055402 (to HAT), P20GM125498 (to SAD), and CDC BAA 2017-N-18041 (to AMDS). Mouse experiments were supported by start-up funds from the UNC Lineberger Comprehensive Cancer Center (to HML). The Zika-TS cohort in 749 León, Nicaragua was supported with NIH grant R21AI129532 (to SBD and FB). At the University of Vermont, cell sorting was performed by the UVM-COM Flow Cytometry and Cell Sorting Facility (with thanks to Roxana del Rio-Guerra) and supported by NIH grant S10-ODO18175 (to Jonathan Boyson) and NIH grant P30 GM118228. Sequencing work was performed by the Vermont Integrated Genomics Resource and supported by NIH grant P30GM118228. NIH contract HHSN272201400058C (to BJD) supported epitope mapping. 
Address correspondence to: Aravinda de Silva, CB 7292, 9024 Burnett Womack, 160 Dental Circle, Chapel Hill, North Carolina 27599-7292, USA. Phone: 919.962.4891; Email: aravinda_desilva@med.unc.edu. Or to: Sean Diehl, Room C205, Given Medical Building, 89 Beaumont Avenue, Burlington, Vermont 05405, USA. Phone: 802.656.9860; Email: sean.diehl@med.uvm.edu.

1. Lazear HM, Diamond MS. Zika virus: New clinical syndromes and its emergence in the western hemisphere. $J$ Virol. 2016;90(10):4864-4875.

2. Fauci AS, Morens DM. Zika virus in the Americas--yet another arbovirus threat. N Engl J Med. 2016;374(7):601-604.

3. Paules CI, Fauci AS. Yellow fever - once again on the radar screen in the Americas. N Engl J Med. 2017;376(15):1397-1399.

4. Collins MH, Metz SW. Progress and works in progress: Update on flavivirus vaccine development. Clin Ther. 2017;39(8):1519-1536.

5. Weiskopf D, Sette A. T-cell immunity to infection with dengue virus in humans. Front Immunol. 2014;5:93.

6. Collins M, de Silva A. Host response: Cross-fit T cells battle Zika virus. Nat Microbiol. 2017;2:17082.

7. Zent O, Hennig R, Banzhoff A, Bröker M. Protection against tick-borne encephalitis with a new vaccine formulation free of protein-derived stabilizers. J Travel Med. 2005;12(2):85-93.

8. Hoke CH, et al. Protection against Japanese encephalitis by inactivated vaccines. N Engl J Med. 1988;319(10):608-614

9. Poland JD, Calisher CH, Monath TP, Downs WG, Murphy K. Persistence of neutralizing antibody 30-35 years after immunization with 17D yellow fever vaccine. Bull World Health Organ. 1981;59(6):895-900.

10. Gotuzzo E, Yactayo S, Córdova E. Efficacy and duration of immunity after yellow fever vaccination: systematic review on the need for a booster every 10 years. Am J Trop Med Hyg. 2013;89(3):434-444.

11. Ng T, Hathaway D, Jennings N, Champ D, Chiang YW, Chu HJ. Equine vaccine for West Nile virus. Dev Biol (Basel). 2003;114:221-227.

12. Wahala WM, Silva AM. The human antibody response to dengue virus infection. Viruses. 2011;3(12):2374-2395.

13. Kirkpatrick BD, et al. Robust and balanced immune responses to all 4 dengue virus serotypes following administration of a single dose of a live attenuated tetravalent dengue vaccine to healthy, flavivirus-naive adults. J Infect Dis. 2015;212(5):702-710.

14. Guy B, Lang J, Saville M, Jackson N. Vaccination against dengue: Challenges and current developments. Annu Rev Med. 2016;67:387-404.

15. Modis Y, Ogata S, Clements D, Harrison SC. A ligand-binding pocket in the dengue virus envelope glycoprotein. Proc Natl Acad Sci USA. 2003;100(12):6986-6991.

16. Sirohi D, et al. The 3.8 Å resolution cryo-EM structure of Zika virus. Science. 2016;352(6284):467-470.

17. Kostyuchenko VA, et al. Structure of the thermally stable Zika virus. Nature. 2016;533(7603):425-428.

18. Teoh EP, et al. The structural basis for serotype-specific neutralization of dengue virus by a human antibody. Sci Transl Med. 2012;4(139):139ra83.

19. Gallichotte EN, et al. A new quaternary structure epitope on dengue virus serotype 2 is the target of durable type-specific neutralizing antibodies. MBio. 2015;6(5):e01461-e01415.

20. de Alwis R, et al. Identification of human neutralizing antibodies that bind to complex epitopes on dengue virions. Proc Natl Acad Sci USA. 2012;109(19):7439-7444.

21. Kaufmann B, et al. Neutralization of West Nile virus by cross-linking of its surface proteins with Fab fragments of the human monoclonal antibody CR4354. Proc Natl Acad Sci USA. 2010;107(44):18950-18955

22. Hadjilaou A, Green AM, Coloma J, Harris E. Single-cell analysis of B cell/antibody cross-reactivity using a novel multicolor fluorospot assay. J Immunol. 2015;195(7):3490-3496.

23. Mathew A, et al. B-cell responses during primary and secondary dengue virus infections in humans. J Infect Dis. 2011;204(10):1514-1522.

24. Bhatt S, et al. The global distribution and burden of dengue. Nature. 2013;496(7446):504-507.

25. Musso D, Gubler DJ. Zika virus. Clin Microbiol Rev. 2016;29(3):487-524.

26. Speer SD, Pierson TC. VIROLOGY. Diagnostics for Zika virus on the horizon. Science.2016;353(6301):750-751

27. Premkumar L, et al. Development of envelope protein antigens to serologically differentiate Zika virus infection from dengue virus infection. J Clin Microbiol. 2018;56(3):e01504-17.

28. Rabe IB, et al. Interim guidance for interpretation of Zika virus antibody test results. MMWR Morb Mortal Wkly Rep. 2016;65(21):543-546.

29. Lanciotti RS, et al. Genetic and serologic properties of Zika virus associated with an epidemic, Yap State, Micronesia, 2007. Emerging Infect Dis. 2008;14(8):1232-1239.

30. Halstead SB. Biologic evidence required for Zika disease enhancement by dengue antibodies. Emerging Infect Dis. 2017;23(4):569-573.

31. McCracken MK, et al. Impact of prior flavivirus immunity on Zika virus infection in rhesus macaques. PLoS Pathog. 2017;13(8):e1006487.

32. George J, et al. Prior exposure to Zika virus significantly enhances peak dengue-2 viremia in rhesus macaques. Sci Rep 2017;7(1):10498.

33. Priyamvada L, et al. Human antibody responses after dengue virus infection are highly cross-reactive to Zika virus. Proc Natl Acad Sci USA. 2016;113(28):7852-7857.

34. Robbiani DF, et al. Recurrent potent human neutralizing antibodies to Zika virus in Brazil and Mexico. Cell. 2017;169(4):597-609.e11.

35. Rogers TF, et al. Zika virus activates de novo and cross-reactive memory B cell responses in dengue-experienced donors. Sci Immunol. 2017;2(14):eaan6809.

36. Stettler K, et al. Specificity, cross-reactivity, and function of antibodies elicited by Zika virus infection. Science. 2016;353(6301):823-826. 
37. Montoya M, et al. Longitudinal analysis of antibody cross-neutralization following Zika virus and dengue virus infection in Asia and the Americas. J Infect Dis. 2018;218(4):536-545.

38. Collins $\mathrm{MH}$, et al. Lack of durable cross-neutralizing antibodies against Zika virus from dengue virus infection. Emerging Infect Dis. 2017;23(5):773-781.

39. Kwakkenbos MJ, et al. Generation of stable monoclonal antibody-producing B cell receptor-positive human memory B cells by genetic programming. Nat Med. 2010;16(1):123-128.

40. Smith K, et al. Rapid generation of fully human monoclonal antibodies specific to a vaccinating antigen. Nat Protoc. 2009;4(3):372-384.

41. Ho IY, et al. Refined protocol for generating monoclonal antibodies from single human and murine B cells. J Immunol Methods. 2016;438:67-70.

42. Dejnirattisai W, et al. A new class of highly potent, broadly neutralizing antibodies isolated from viremic patients infected with dengue virus. Nat Immunol. 2015;16(2):170-177.

43. Wang J, et al. A human bi-specific antibody against Zika virus with high therapeutic potential. Cell. 2017;171(1):229-241.e15.

44. Lazear HM, et al. A mouse model of Zika virus pathogenesis. Cell Host Microbe. 2016;19(5):720-730.

45. Davidson E, Doranz BJ. A high-throughput shotgun mutagenesis approach to mapping B-cell antibody epitopes. Immunology. 2014;143(1):13-20.

46. Sapparapu G, et al. Neutralizing human antibodies prevent Zika virus replication and fetal disease in mice. Nature. 2016;540(7633):443-447.

47. Fibriansah $\mathrm{G}$, et al. A highly potent human antibody neutralizes dengue virus serotype 3 by binding across three surface proteins. Nat Commun. 2015;6:6341.

48. Kiermayr S, Stiasny K, Heinz FX. Impact of quaternary organization on the antigenic structure of the tick-borne encephalitis virus envelope glycoprotein E. J Virol. 2009;83(17):8482-8491.

49. Wang Q, et al. Molecular determinants of human neutralizing antibodies isolated from a patient infected with Zika virus. Sci Transl Med. 2016;8(369):369ra179.

50. Yu L, et al. Delineating antibody recognition against Zika virus during natural infection. JCI Insight. 2017;2(12):93042.

51. Williams KL, Wahala WM, Orozco S, de Silva AM, Harris E. Antibodies targeting dengue virus envelope domain III are not required for serotype-specific protection or prevention of enhancement in vivo. Virology. 2012;429(1):12-20.

52. Mathew A, et al. B-cell responses during primary and secondary dengue virus infections in humans. J Infect Dis. 2011;204(10):1514-1522.

53. Lai L, et al. Innate, T-, and B-cell responses in acute human Zika patients. Clin Infect Dis. 2018;66(1):1-10

54. Wagner SD, Milstein C, Neuberger MS. Codon bias targets mutation. Nature. 1995;376(6543):732.

55. Jones PT, Dear PH, Foote J, Neuberger MS, Winter G. Replacing the complementarity-determining regions in a human antibody with those from a mouse. Nature. 1986;321(6069):522-525.

56. Amit AG, Mariuzza RA, Phillips SE, Poljak RJ. Three-dimensional structure of an antigen-antibody complex at 2.8 A resolution. Science. 1986;233(4765):747-753.

57. Rajewsky K. Clonal selection and learning in the antibody system. Nature. 1996;381(6585):751-758.

58. Klein F, et al. Somatic mutations of the immunoglobulin framework are generally required for broad and potent HIV-1 neutralization. Cell. 2013;153(1):126-138.

59. Goo L, et al. The Zika virus envelope protein glycan loop regulates virion antigenicity. Virology. 2018;515:191-202.

60. Sessions OM, et al. Analysis of dengue virus genetic diversity during human and mosquito infection reveals genetic constraints. PLoS Negl Trop Dis. 2015;9(9):e0004044.

61. Balmaseda A, et al. Antibody-based assay discriminates Zika virus infection from other flaviviruses. Proc Natl Acad Sci USA. 2017;114(31):8384-8389.

62. Dowd KA, et al. Broadly neutralizing activity of Zika virus-immune sera identifies a single viral serotype. Cell Rep. 2016;16(6):1485-1491.

63. Aliota MT, et al. Heterologous protection against Asian Zika virus challenge in rhesus macaques. PLoS Negl Trop Dis. 2016;10(12):e0005168.

64. Smith SA, Zhou Y, Olivarez NP, Broadwater AH, de Silva AM, Crowe JE. Persistence of circulating memory B cell clones with potential for dengue virus disease enhancement for decades following infection. $J$ Virol. 2012;86(5):2665-2675.

65. Beltramello M, et al. The human immune response to Dengue virus is dominated by highly cross-reactive antibodies endowed with neutralizing and enhancing activity. Cell Host Microbe. 2010;8(3):271-283.

66. Katzelnick LC, et al. Antibody-dependent enhancement of severe dengue disease in humans. Science. 2017;358(6365):929-932

67. Dejnirattisai W, et al. Dengue virus sero-cross-reactivity drives antibody-dependent enhancement of infection with Zika virus. Nat Immunol. 2016;17(9):1102-1108.

68. Castanha PMS, et al. Dengue virus-specific antibodies enhance Brazilian Zika virus infection. J Infect Dis. 2017;215(5):781-785.

69. Paul LM, et al. Dengue virus antibodies enhance Zika virus infection. Clin Transl Immunology. 2016;5(12):e117.

70. Bardina SV, et al. Enhancement of Zika virus pathogenesis by preexisting antiflavivirus immunity. Science. 2017;356(6334):175-180.

71. Pantoja P, et al. Zika virus pathogenesis in rhesus macaques is unaffected by pre-existing immunity to dengue virus. Nat Commun. 2017;8:15674.

72. Halai UA, et al. Maternal Zika virus disease severity, virus load, prior dengue antibodies, and their relationship to birth outcomes. Clin Infect Dis. 2017;65(6):877-883.

73. Kawiecki AB, Christofferson RC. Zika virus-induced antibody response enhances dengue virus serotype 2 replication in vitro. J Infect Dis. 2016;214(9):1357-1360.

74. Dick GW, Kitchen SF, Haddow AJ. Zika virus. I. Isolations and serological specificity. Trans R Soc Trop Med Hyg. 1952;46(5):509-520.

75. Haddow AD, et al. Genetic characterization of Zika virus strains: geographic expansion of the Asian lineage. PLoS Negl Trop Dis. 2012;6(2):e1477.

76. Baronti C, Piorkowski G, Charrel RN, Boubis L, Leparc-Goffart I, de Lamballerie X. Complete coding sequence of Zika virus 
from a French Polynesia outbreak in 2013. Genome Announc. 2014;2(3):e00500-14.

77. Lanciotti RS, Lambert AJ, Holodniy M, Saavedra S, Signor Ldel C. Phylogeny of Zika virus in western hemisphere, 2015. Emerging Infect Dis. 2016;22(5):933-935.

78. Diehl SA, et al. STAT3-mediated up-regulation of BLIMP1 Is coordinated with BCL6 down-regulation to control human plasma cell differentiation. J Immunol. 2008;180(7):4805-4815.

79. Nivarthi UK, et al. Mapping the human memory B cell and serum neutralizing antibody responses to dengue virus serotype 4 infection and vaccination. J Virol. 2017;91(5):e02041-16.

80. Swanstrom JA, et al. Dengue virus envelope dimer epitope monoclonal antibodies isolated from dengue patients are protective against Zika virus. MBio. 2016;7(4):01123-16.

81. Henchal EA, Gentry MK, McCown JM, Brandt WE. Dengue virus-specific and flavivirus group determinants identified with monoclonal antibodies by indirect immunofluorescence. Am J Trop Med Hyg. 1982;31(4):830-836.

82. Metz SW, et al. In vitro assembly and stabilization of dengue and Zika virus envelope protein homo-dimers. Sci Rep. 2017;7(1):4524.

83. Metz SW, et al. Dengue virus-like particles mimic the antigenic properties of the infectious dengue virus envelope. Virol $J$. 2018;15(1):60

84. Bogan AA, Thorn KS. Anatomy of hot spots in protein interfaces. J Mol Biol. 1998;280(1):1-9.

85. Lo Conte L, Chothia C, Janin J. The atomic structure of protein-protein recognition sites. J Mol Biol. 1999;285(5):2177-2198. 